\title{
Serum Butyrylcholinesterase Activity: A Biomarker for Parkinson's Disease and Related Dementia
}

\author{
Mei-Xue Dong, ${ }^{1}$ Xiao-Min $\mathrm{Xu},{ }^{1}$ Ling $\mathrm{Hu},{ }^{2}$ Yang Liu, ${ }^{1}$ \\ Yuan-Jun Huang, ${ }^{1}$ and You-Dong Wei ${ }^{1}$ \\ ${ }^{1}$ Department of Neurology, The First Affiliated Hospital of Chongqing Medical University, Chongqing, China \\ ${ }^{2}$ Department of Neurology, Chongqing Fifth People's Hospital, Chongqing, China
}

Correspondence should be addressed to You-Dong Wei; youdongwei1966@163.com

Received 29 March 2017; Revised 13 June 2017; Accepted 4 July 2017; Published 3 August 2017

Academic Editor: Cristiano Capurso

Copyright (C) 2017 Mei-Xue Dong et al. This is an open access article distributed under the Creative Commons Attribution License, which permits unrestricted use, distribution, and reproduction in any medium, provided the original work is properly cited.

\begin{abstract}
Objective. This study aim to determine changes of serum butyrylcholinesterase (BChE) activity in PD patients and related dementia. Patients and Methods. Consecutive PD patients and healthy controls were included and clinical data were collected. Fast serum BChE activity was determined and compared between healthy controls and PD patients. Independent risk factors were performed for BChE activity, PD, and related dementia. The relationship between BChE activity and disease severity was also evaluated. Receiver operating characteristic (ROC) curves were obtained to explore serum BChE activity in distinguishing PD patients and related dementia. Results. Serum BChE activity mainly independently correlated with gender, albumin, triglyceride, body mass index, and PD. Serum BChE activity decreased in PD patients compared with healthy controls. Based on the ROC curve, the optimal cut-off point was $6864.08 \mathrm{IU} / \mathrm{L}$ for distinguishing PD patients, and the sensitivity and specificity values were $61.8 \%$ and 72.1\%. It inversely correlated with Unified Parkinson's Disease Rating Scale score. BChE activity decreased in PD-related dementia compared with those without dementia. The sensitivity and specificity values were $70.6 \%$ and $76.3 \%$, respectively, with an optimal cut-off point of $6550.00 \mathrm{IU} / \mathrm{L}$. Conclusions. Serum BChE activity can be regarded as a biomarker for PD and related dementia.
\end{abstract}

\section{Introduction}

Parkinson's disease (PD), the second most common neurodegenerative disease following Alzheimer's disease, afflicts about $1 \%$ of the elderly over 60 years old and $3 \%$ of those over 80 years old [1]. Although extensive studies have been performed to identify PD biomarkers, no validated biomarkers have been found to date and the diagnosis remains based primarily on clinical symptoms. PD is a complicated interplay of genetics and the environment, typically presenting as death of dopaminergic neurons in the substantia nigra pars compacta [2]. However, it also involves alterations of neurotransmitters other than dopamine and regions of the nervous system outside the substantia nigra [2]. Those various pathological changes result in many other nonmotor symptoms, including olfactory dysfunction, cognitive impairment, depression, anxiety, sleep disorders, autonomic dysfunction, pain, and fatigue.

Cognitive impairment is common in PD patients and is associated with age, disease duration, and disease severity.
It can also develop in PD patients following treatment with anticholinergic drugs, such as benzhexol and benztropine. More than $80 \%$ of $\mathrm{PD}$ patients with cognitive impairment develop dementia over a long-term disease duration [3]. PD patients with dementia bring particular challenges to patient management, which further aggravates functional impairments caused by motor symptoms [4]. Patients with dementia have greater requirements of families and caregivers in terms of activities of daily living, resulting in a social-psychological strain for both PD patients and their families. Early dementia treatment would be more effective [5]. Hence, there is an urgent need to identify potential biomarkers for Parkinson's disease and related dementia.

With exception of changes in dopaminergic neurons in the nigrostriatal pathway, a former PET study showed that PD patients with dementia also had suffered from severe cholinergic deficits all over the brain [6]. Acetylcholine (Ach) is the main parasympathetic neurotransmitter released by cholinergic neurons. It is a key contributor to stress and enhances 
neuronal excitability. In addition, it also potently modulates the classical immune response by activating $\alpha 7$ nicotinic Ach receptor on the surface of leukocytes in the so-called "cholinergic anti-inflammatory response" [7]. The cholinergic system is involved in many physiological functions of the central nervous system, and Ach can be hydrolyzed by acetylcholinesterase (AChE) and butyrylcholinesterase (BChE), two independent enzymes in the circulation system. Serum BChE is mainly synthesized in the liver and can be clinically used as a marker of organophosphate poisoning [8]. However, increasing evidence suggests that BChE activity is also associated with disease severity and can be used to predict prognosis in many diseases [7]. Although AChE activity reportedly decreases in Parkinson's disease [9], the relationship between $\mathrm{BChE}$ activity and Parkinson's disease had not been clearly performed.

Serum is a relatively noninvasive and reliable clinical specimen. The present study aimed to determine changes of serum BChE activity in PD patients and related dementia.

\section{Materials and Methods}

2.1. Patients. A total of 84 consecutive patients with Parkinson's disease were included in accordance with the following inclusion criteria from the Department of Neurology of the First Affiliated Hospital of Chongqing Medical University from April 2015 to December 2016. In all, eight patients were excluded per the exclusion criteria. The inclusion criteria were as follows: (i) Parkinson's disease diagnosed according to the recommendation from EFNS and MDS-ES [10]; (ii) patients treated without any drugs with a known effect on cholinesterase (ChE) activity (including anticholinergic drugs benzhexol, benztropine, atropine, anisodarnine, and scopolamine; ChE inhibitors donepezil, galantamine, and rivastigmine; statins; acetylsalicylic acid; isosorbide-based drugs; fluoxetine, sertraline, and amitriptyline; benzodiazepine derivates; itopride; chlordiazepoxide; histamine 2 receptor antagonist; or Ginkgo extract) [11]. Exclusion criteria were as follows: (i) secondary parkinsonian disorders or parkinsonism-plus syndrome; (ii) coexistence of severe systemic diseases (e.g., tumor, chronic heart failure, chronic obstructive pulmonary disease, hepatitis, and nephritis) or infectious diseases at the time of enrolment; (iii) history of stroke, brain surgery or injury, Alzheimer's disease, motor neuron disease, or other central nervous system diseases $[12,13]$.

2.2. Healthy Controls. A total of 61 healthy controls were included at the same time from the Department of Physical Examination. The controls were without history of stroke, brain surgery or injury, neurodegenerative disease, or infectious disease. This study was approved by the ethics committee of the First Affiliated Hospital of Chongqing Medical University and performed in accordance with ethical principles. All subjects provided written informed consent prior to inclusion in this study.

2.3. Clinical Characteristics. Clinical data from patients and healthy controls were collected. Fast serum samples were obtained by centrifugation at $2000 \times \mathrm{g}$ for $10 \mathrm{~min}$ at $4^{\circ} \mathrm{C}$ after being collected at 6:00 am by puncture of the median cubital vein. Then, levels of albumin, alanine transaminase (ALT), aspartate aminotransferase (AST), total cholesterol (TC), triglyceride (TG), and BChE activity were measured soon using a Cobas Integra 400 plus automatic biochemical analyser with matched reagent kits (Roche, Basel, Switzerland). Serum BChE activity was determined using Cobas Integra Cholinesterase kit (20737380; Roche) and butyrylthiocholine served as the substrate. Other laboratory benchmarks were quantified per manufacture specifications.

2.4. Disease Assessment Scale. Unified Parkinson's disease rating scale (UPDRS), modified Hoehn-Yahr staging scale, and Mini-Mental State Examination (MMSE) scores were assessed at the time blood specimens were collected. Total UPDRS scores were used to assess disease severity of PD. Modified Hoehn-Yahr staging scale scores were used to group PD patients into early stage $(<2.5)$ or advanced stage $(\geq 2.5)$ [14]. MMSE scores were used to detect cognitive impairment in PD patients, and three different cut-off points were used to determine dementia according to patient education level [15]. All scales were independently assessed by two observers.

2.5. Statistical Methods. Statistical analyses were performed using a commercially available software package (IBM SPSS version 22.0, Armonk, NY, USA). Some statistical diagrams were produced using GraphPad Prism 5 (Version 5.01, GraphPad Software, La Jolla, CA, USA). Clinical characteristics were compared between healthy controls and PD patients, with or without dementia, using Pearson $\chi^{2}$-tests or Fisher exact tests for categorical data and Student's $t$-tests for continuous data. Categorical data were exhibited as absolute numbers and percentage (\%), while continuous data were exhibited as mean values \pm standard deviation. Univariate and multivariate linear regression analyses were performed to determine independent correlation factors for $\mathrm{BChE}$ activity. Univariate and multivariate binary logistic regression analyses were performed to determine independent correlation factors for PD and related dementia. Pearson correlation coefficients were obtained and scatter diagrams were plotted to explore the relationships between BChE activity and UPDRS scores or MMSE scores. To assess the reliability of serum BChE activity in discriminating $\mathrm{PD}$ or related dementia, receiver operating characteristic (ROC) curves were plotted and the areas under the curve (AUCs) were estimated. Based on ROC curves, the sensitivity and specificity values were calculated according to optimal cutoff points. Tests were two-tailed and significance levels were chosen as $P<0.05$.

\section{Results}

3.1. Clinical Characteristics of PD Patients and Healthy Controls. All included patients had been diagnosed prior to the study and were treated with dopamine analogues or dopamine receptor agonists at the time of enrolment. Clinical characteristics of PD patients and healthy controls are presented in Table 1. There were no significant differences 
TABLE 1: Clinical characteristics of PD patients and healthy controls.

\begin{tabular}{lccc}
\hline Variable (SD/\%) & PD (76) & Control (61) & $P$ value \\
\hline Age (year) & $69.30 \pm 8.99$ & $67.87 \pm 5.74$ & 0.260 \\
Gender, male & $45(59.2 \%)$ & $29(47.5 \%)$ & $39.92 \pm 3.52$ \\
Albumin (g/L) & $38.99 \pm 3.93$ & $20.03 \pm 9.94$ & 0.173 \\
ALT (U/L) & $18.99 \pm 13.58$ & $20.93 \pm 6.51$ & 0.152 \\
AST (U/L) & $23.04 \pm 13.59$ & $4.19 \pm 0.91$ & 0.238 \\
TC (mmol/L) & $4.25 \pm 0.84$ & $1.45 \pm 0.79$ & 0.701 \\
TG (mmol/L) & $1.25 \pm 0.76$ & $23.71 \pm 3.16$ & 0.116 \\
BMI & $23.28 \pm 3.36$ & $20(32.8 \%)$ & 0.449 \\
Smoking history & $14(18.2 \%)$ & $5(7.8 \%)$ & 0.053 \\
Alcohol consumption & $7(9.2 \%)$ & $7(10.9 \%)$ & 0.835 \\
CHD & $15(19.7 \%)$ & $29(47.5 \%)$ & 0.191 \\
Hypertension & $29(38.2 \%)$ & $10(16.4 \%)$ & 0.269 \\
Diabetes mellitus & $10(13.2 \%)$ & $9(14.8 \%)$ & 0.594 \\
Hypercholesterolaemia & $15(19.7 \%)$ & $7904.29 \pm 1470.77$ \\
BChE activity (IU/L) & $6869.94 \pm 1343.51$ & 0.446 \\
\hline
\end{tabular}

PD, Parkinson's disease; SD, standard deviation; ALT, alanine transaminase; AST, aspartate aminotransferase; TC, total cholesterol; TG, triglyceride; BMI, body mass index; CHD, coronary heart disease; BChE, butyrylcholinesterase.

TABLE 2: Univariate and multivariate linear regression analysis of correlation factors for serum butyrylcholinesterase activity.

\begin{tabular}{|c|c|c|c|c|}
\hline \multirow{2}{*}{ Variable } & \multicolumn{2}{|c|}{ Univariate } & \multicolumn{2}{|c|}{ Multivariate } \\
\hline & Beta value & $P$ value & Beta value & $P$ value \\
\hline Age & -0.264 & 0.002 & - & - \\
\hline Gender & 0.258 & 0.002 & 0.183 & 0.006 \\
\hline Albumin & 0.477 & 0.000 & 0.350 & 0.000 \\
\hline ALT & -0.014 & 0.867 & I & / \\
\hline AST & -2.052 & 0.042 & - & - \\
\hline $\mathrm{TC}$ & 3.876 & 0.000 & - & - \\
\hline TG & 0.439 & 0.000 & 0.235 & 0.001 \\
\hline BMI & 0.322 & 0.000 & 0.163 & 0.020 \\
\hline Smoking history & -0.028 & 0.744 & / & l \\
\hline Alcohol consumption & -0.001 & 0.991 & / & / \\
\hline Hypertension & 0.074 & 0.392 & l & l \\
\hline Diabetes mellitus & 0.059 & 0.496 & I & / \\
\hline Hypercholesterolaemia & 0.166 & 0.052 & - & - \\
\hline CHD & -0.090 & 0.297 & I & l \\
\hline $\mathrm{PD}$ & -0.347 & 0.000 & -0.240 & 0.000 \\
\hline
\end{tabular}

Beta value, adjusted regression coefficient; ALT, alanine transaminase; AST, aspartate aminotransferase; TC, total cholesterol; TG, triglyceride; BMI, body mass index; $\mathrm{CHD}$, coronary heart disease; PD, Parkinson's disease.

For the multivariate linear regression analysis using a backward method based on the results from univariate linear regression analysis, the values were "/" if variables were excluded before the analysis, and the values were "-" if variables were excluded after the analysis.

between these two groups in age, gender, or any other characteristics. Serum BChE activity in PD patients were lower than in healthy controls. PD patients had a slightly lower percentage of smoking history than healthy controls. According to the linear regression analyses presented in Table 2, gender, albumin, TG, BMI, and PD were all independent correlation factors for serum BChE activity while smoking history was not. For PD, only smoking history and BChE activity were independent correlation factors based on binary logistic regression analyses (Table 3). To further confirm whether smoking history affected BChE activity in the two groups, a subgroup analysis was performed and is shown in Figure 1. There was no significant difference in serum BChE activity between PD patients with and without smoking history. The ROC curve of serum BChE activity for detecting PD patients is presented in Figure 2(a) and the AUC for PD was 0.699. Based on the ROC curve and diagnostic value of the different cut-off points, the optimal cut-off point, where the sensitivity value plus the specificity value was maximum, was found to be 6864.08 IU/L. The sensitivity and specificity values of serum BChE activity for detecting PD were $61.8 \%$ and $72.1 \%$, respectively. 
TABLE 3: Univariate and multivariate binary logistic regression analysis of correlation factors for Parkinson's disease.

\begin{tabular}{|c|c|c|c|c|}
\hline \multirow{2}{*}{ Variable } & \multicolumn{2}{|c|}{ Univariate } & \multicolumn{2}{|c|}{ Multivariate } \\
\hline & OR (LL UL) & $P$ value & OR (LL UL) & $P$ value \\
\hline Age & $1.025(0.980 \sim 1.071)$ & 0.281 & I & / \\
\hline Gender & $0.624(0.316 \sim 1.232)$ & 0.174 & / & / \\
\hline Albumin & $0.935(0.852 \sim 1.026)$ & 0.154 & I & l \\
\hline ALT & $1.004(0.988 \sim 1.021)$ & 0.622 & I & I \\
\hline AST & $1.022(0.990 \sim 1.054)$ & 0.175 & I & I \\
\hline TC & $1.085(0.718 \sim 1.639)$ & 0.699 & I & I \\
\hline TG & $0.700(0.445 \sim 1.100)$ & 0.121 & / & I \\
\hline BMI & $0.960(0.866 \sim 1.066)$ & 0.446 & I & I \\
\hline Smoking history & $0.463(0.210 \sim 1.019)$ & 0.056 & $0.404(0.175 \sim 0.936)$ & 0.034 \\
\hline Alcohol consumption & $0.414(0.152 \sim 1.128)$ & 0.085 & I & I \\
\hline Hypertension & $0.681(0.344 \sim 1.348)$ & 0.270 & I & I \\
\hline Diabetes mellitus & $0.773(0.299 \sim 1.997)$ & 0.595 & / & I \\
\hline Hypercholesterolaemia & $1.421(0.575 \sim 3.513)$ & 0.447 & I & I \\
\hline $\mathrm{CHD}$ & $1.897(0.720 \sim 4.999)$ & 0.195 & I & I \\
\hline BChE activity & $0.999(0.999 \sim 1.000)$ & 0.000 & $0.999(0.999 \sim 1.000)$ & 0.000 \\
\hline
\end{tabular}

OR, odds ratio; LL, lower limit; UL, upper limit; ALT, alanine transaminase; AST, aspartate aminotransferase; TC, total cholesterol; TG, triglyceride; BMI, body mass index; CHD, coronary heart disease; BChE, butyrylcholinesterase.

For the multivariate binary logistic regression analysis using a conditional backward method based on the results from univariate binary logistic regression analysis, the values were " $"$ " if variables were excluded before the analysis.

TABLE 4: Univariate and multivariate linear regression analysis of correlation factors for serum butyrylcholinesterase activity in patient with Parkinson's disease.

\begin{tabular}{|c|c|c|c|c|}
\hline \multirow{2}{*}{ Variable } & \multicolumn{2}{|c|}{ Univariate } & \multicolumn{2}{|c|}{ Multivariate } \\
\hline & Beta value & $P$ value & Beta value & $P$ value \\
\hline Disease duration & -0.202 & 0.080 & I & / \\
\hline Age & -0.354 & 0.002 & - & - \\
\hline Gender & 0.248 & 0.031 & 0.277 & 0.001 \\
\hline Albumin & 0.568 & 0.000 & 0.493 & 0.000 \\
\hline ALT & 0.029 & 0.802 & l & l \\
\hline AST & -0.196 & 0.089 & l & l \\
\hline $\mathrm{TC}$ & 0.399 & 0.000 & - & - \\
\hline TG & 0.261 & 0.023 & - & - \\
\hline BMI & 0.305 & 0.007 & 0.188 & 0.029 \\
\hline Smoking history & 0.001 & 0.993 & l & l \\
\hline Alcohol consumption & -0.051 & 0.662 & 1 & l \\
\hline Hypertension & 0.024 & 0.834 & I & l \\
\hline Diabetes mellitus & 0.076 & 0.515 & I & l \\
\hline Hypercholesterolaemia & 0.133 & 0.251 & I & l \\
\hline $\mathrm{CHD}$ & -0.097 & 0.406 & 1 & l \\
\hline Advanced stage & -0.190 & 0.101 & I & I \\
\hline Dementia & -0.360 & 0.001 & -0.319 & 0.000 \\
\hline
\end{tabular}

Beta value, adjusted regression coefficient; ALT, alanine transaminase; AST, aspartate aminotransferase; TC, total cholesterol; TG, triglyceride; BMI, body mass index; CHD, coronary heart disease; PD, Parkinson's disease.

For the multivariate linear regression analysis using a backward method based on the results from univariate linear regression analysis, the values were " $"$ " if variables were excluded before the analysis, and the values were "-" if variables were excluded after the analysis.

3.2. Disease Severity of PD Patients. For PD patients, serum BChE activity inversely correlated with UPDRS scores $(r=$ $-0.404, P<0.001)$. Relationship between BChE activity and UPDRS score is presented in Figure 2(b).
3.3. Clinical Correlation Factors for BChE Activity in PD Patients. Results of univariate and multivariate linear regression analysis for serum BChE activity in PD patients are shown in Table 4. Gender, albumin, BMI, and dementia were 
TABLE 5: Univariate linear regression analysis of anti-Parkinson's disease drugs for serum butyrylcholinesterase activity in PD patient.

\begin{tabular}{|c|c|c|c|}
\hline Variable & Patient percentage & Beta value & $P$ value \\
\hline Total patients & $76(100 \%)$ & I & / \\
\hline Levodopa and Benserazide Hydrochloride Tablet & $54(71.1 \%)$ & -0.169 & 0.143 \\
\hline Carbidopa and Levodopa-CR Tablet & $8(10.5 \%)$ & -0.106 & 0.362 \\
\hline Levodopa Tablet & $1(1.3 \%)$ & 0.042 & 0.717 \\
\hline Piribedil-SR Tablet & $27(35.5 \%)$ & 0.759 & 0.450 \\
\hline Pramipexole Dihydrochloride Tablet & $15(19.7 \%)$ & -0.042 & 0.720 \\
\hline
\end{tabular}

PD, Parkinson's disease; Beta value, adjusted regression coefficient; CR, controlled release; SR, sustained release.

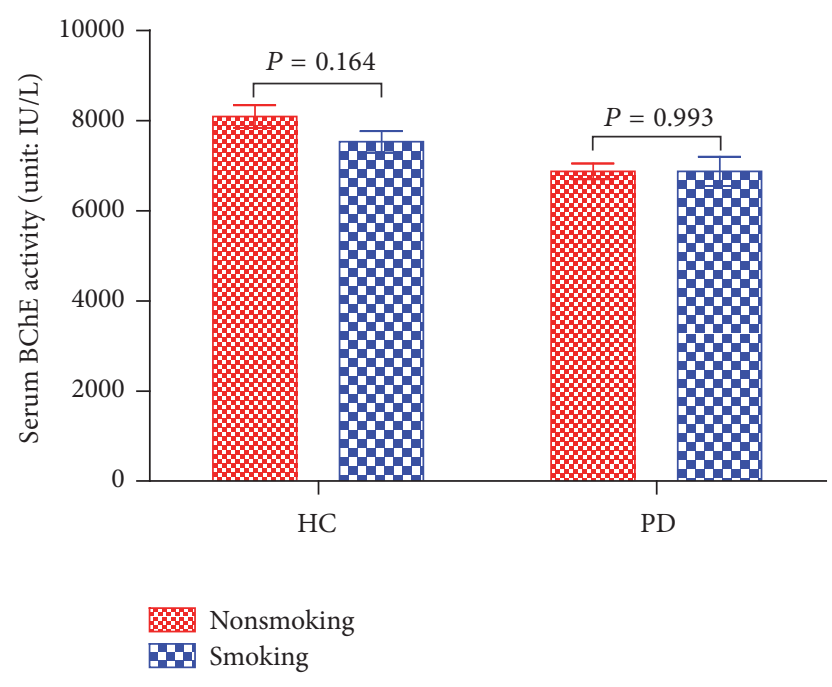

Figure 1: Mean serum BChE activities of different subgroups based on smoking history. No significant difference can be found between smoking and nonsmoking patients in the two groups $(P>0.05)$. BChE, butyrylcholinesterase; HC, healthy control; PD, Parkinson's disease.

all independent correlation factors for serum BChE activity. BChE activity positively correlated with MMSE score $(r=$ $0.359, P=0.001)$ and the relationship is presented in Figure 2(c). The medication status of these patients is presented in Table 5, and no significant drug influence can be found on serum BChE activity based on the univariate linear regression analyses.

3.4. BChE Activity in PD-Related Dementia. PD patients were categorized into two subgroups (with dementia and without dementia) per MMSE scores and education background. Clinical characteristics of these two subgroups are shown in Table 6. The table indicates that patients with dementia were older, had significantly longer disease duration, and presented with a more advanced stage. Patients with dementia had lower BChE activity than patients without dementia. However, according to binary logistic regression analysis, only advanced stage and BChE activity were independent correlation factors for PD-related dementia (Table 7). The ROC curve of serum BChE activity for patients with dementia is shown in Figure 2(d), and the AUC for patients with dementia was 0.752 . Based on the ROC, the sensitivity and specificity values of serum BChE activity for diagnosing
PD-related dementia were $70.6 \%$ and $76.3 \%$, respectively, where the optimal cut-off point was $6550.00 \mathrm{IU} / \mathrm{L}$.

\section{Discussion}

Ach is the main parasympathetic neurotransmitter and can be hydrolyzed by $\mathrm{AChE}$ and $\mathrm{BChE}$, two enzymes in the circulation system. BChE accounts for up to $90 \%$ total serum ChE while its activity is 20 -fold lower than AChE in hydrolyzing Ach [16]. Serum BChE activity has been broadly used as a biomarker for various diseases [17, 18], although its function has not been definitely clarified. In the clinic, it is most widely used for the assessment of organophosphate poisoning [8]. Organophosphates, as well as other forms of environmental toxin exposure, are risk factors for Parkinson's disease [9]. Serum BChE activity in Parkinson's disease has not yet been clearly determined, and we report it for the first time.

Results from this study showed significantly less BChE activity in PD patients compared with healthy controls while the other clinical characteristics showed no significant differences, suggesting the comparison was reasonable. $\mathrm{PD}$ patients usually have a number of comorbidities and $\mathrm{BChE}$ activity is reportedly related to many pathologies, including hepatic function [17] and metabolic syndrome (diabetes mellitus, hypercholesterolemia, TC, and TG) [11, 19]. Hence, linear regression analyses were performed, and $\mathrm{PD}$ was also found to be an independent correlation factor for BChE activity in our study, along with gender, albumin, TG, and BMI [11]. Binary logistic regression analyses were also performed for $\mathrm{PD}$ and determined smoking history and BChE activity as the independent correlation factors. Meanwhile, PD patients had a slightly lower percentage of smoking history compared with healthy controls, which was consistent with a former study [20]. Then a subgroup analysis found no alterations between patients with and without smoking history, suggesting that only $\mathrm{BChE}$ activity correlated with Parkinson's disease independent of hepatic function, smoking history, metabolic syndrome, or any other clinical characteristics.

However, the declined BChE activity was inconsistent with a former study [9], which indicated that BChE activity was not significantly altered in Israeli Parkinson's disease patients compared with younger healthy controls. Their result was controversial as some researchers showed an agedependant difference in BChE activity [11,21], which was also confirmed from our study. Furthermore, serum BChE activity had reportedly decreased in the organophosphates 


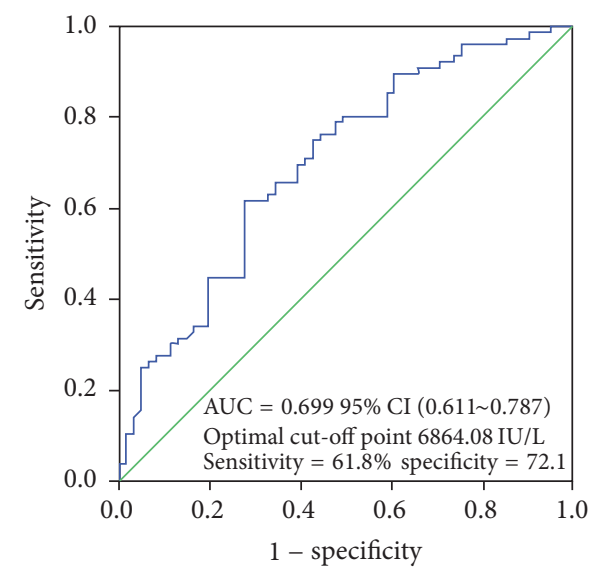

(a)

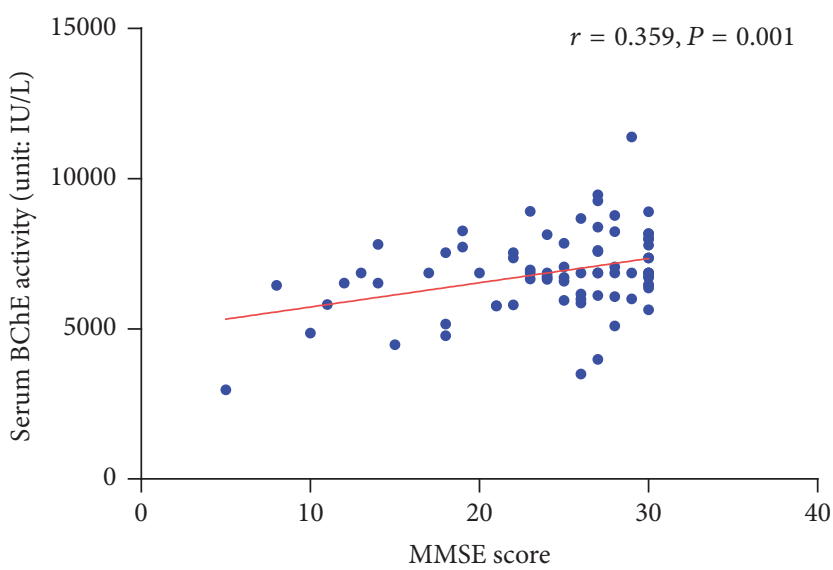

(c)

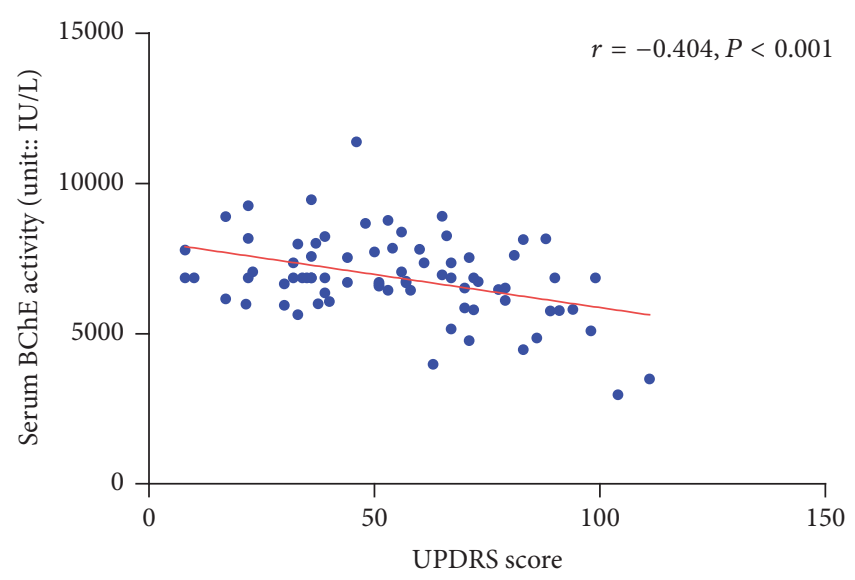

(b)

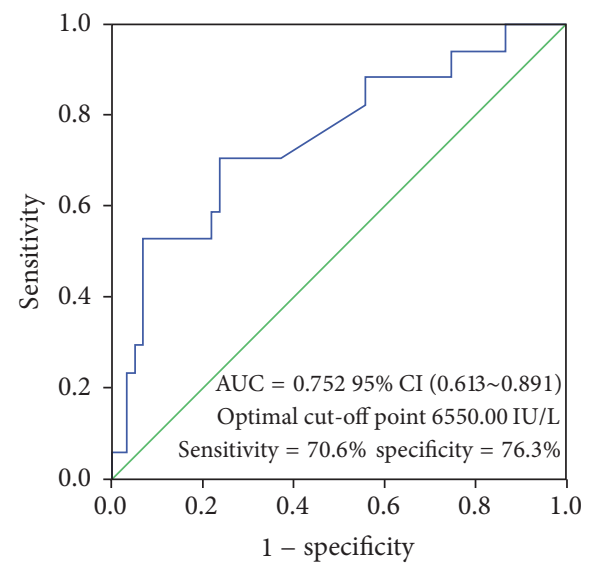

(d)

Figure 2: (a) ROC curve of serum BChE activities for detecting PD patients. (b) A linear correlation is observed between BChE activities and UPDRS scores. (c) A linear correlation is observed between BChE activities and MMSE scores. (d) ROC curve of serum BChE activities for detecting PD patients with dementia. ROC, receiver operating characteristic; BChE, butyrylcholinesterase; PD, Parkinson's disease; AUC, area under the curve; CI, confidence interval; UPDRS, Unified Parkinson's Disease Rating Scale; MMSE, Mini-Mental State Examination.

exposed population [22, 23]. However, nearly $37.6 \%$ of their PD patients were exposed to relatively high levels of organophosphates but without a decreased BChE activity. The inconformity may result from various genetic backgrounds of PD patients in different races. The AUC of ROC for Parkinson's disease in our study was 0.699, and the sensitivity and specificity values of serum BChE activity for diagnosing PD were $61.8 \%$ and $72.1 \%$, respectively, with an optimal cut-off point of $6864.08 \mathrm{IU} / \mathrm{L}$.

UPDRS consists of four assessments and is the most widely used clinical rating scale for evaluating disease severity in Parkinson's disease [24]. We found that BChE activity inversely correlated with UPDRS scores, suggesting that it could serve as a biomarker for evaluating disease severity in PD patient. Mini-Mental State Examination is the most popular scale to determine possible dementia and a positive correlation was also found between serum BChE activity and MMSE score.

In $\mathrm{PD}$ patients, we found that dementia as well as gender, albumin, and BMI, was an independent correlation factor for BChE activity. As all the patients were treated with dopamine analogues or dopamine receptor agonists at the time of enrolment, linear regression analyses were performed to exclude medication influence on BChE activity. Meanwhile, no significant results can be found. Results also showed that PD with dementia had a longer disease duration than PD without dementia, with no significant difference in onset age between these two groups, which was likely due to the face that dementia developed in most PD patients after a long disease duration [3]. These patients, therefore, were at a more advanced stage with more severe clinical symptoms. Additionally, PD patients with dementia had more difficulties in activities of daily living and required more assistance. Thus, their nutritional state was poorer, and $\mathrm{BChE}$ activity was lower than patients without dementia. Logistic regression analysis then confirmed that both $\mathrm{BChE}$ activity and advanced stage were independent correlation factors for PD-related dementia.

The ROC curve was analyzed for serum BChE activity to detect patients with dementia. The sensitivity and specificity values were $70.6 \%$ and $76.3 \%$, respectively, with an optimal cut-off point of $6550.00 \mathrm{IU} / \mathrm{L}$. The specificity value was high 
TABLE 6: Clinical characteristics of PD patients with and without dementia.

\begin{tabular}{|c|c|c|c|}
\hline Variable (SD) & With dementia (17) & Without dementia (59) & $P$ value \\
\hline Disease duration (year) & $8.71 \pm 6.06$ & $5.10 \pm 4.23$ & 0.032 \\
\hline Age (year) & $73.47 \pm 7.89$ & $68.10 \pm 8.98$ & 0.029 \\
\hline Onset age (year) & $64.76 \pm 12.18$ & $63.00 \pm 9.28$ & 0.523 \\
\hline Gender, male & $9(52.9 \%)$ & $36(61.0 \%)$ & 0.551 \\
\hline Albumin (g/L) & $38.18 \pm 3.91$ & $39.22 \pm 3.94$ & 0.338 \\
\hline $\operatorname{ALT}(\mathrm{U} / \mathrm{L})$ & $15.63 \pm 10.74$ & $19.90 \pm 14.18$ & 0.267 \\
\hline $\operatorname{AST}(\mathrm{U} / \mathrm{L})$ & $23.69 \pm 19.87$ & $22.86 \pm 11.55$ & 0.832 \\
\hline $\mathrm{TC}(\mathrm{mmol} / \mathrm{L})$ & $4.05 \pm 0.77$ & $4.31 \pm 0.85$ & 0.272 \\
\hline $\mathrm{TG}(\mathrm{mmol} / \mathrm{L})$ & $1.13 \pm 0.75$ & $1.27 \pm 0.76$ & 0.502 \\
\hline Smoking history & $4(23.5 \%)$ & $10(16.9 \%)$ & 0.794 \\
\hline Alcohol consumption & $3(17.6 \%)$ & $4(6.8 \%)$ & 0.374 \\
\hline Hypertension & $5(29.4 \%)$ & $24(40.7 \%)$ & 0.399 \\
\hline Diabetes mellitus & $2(11.8 \%)$ & $8(13.6 \%)$ & 1.000 \\
\hline Hypercholesterolaemia & $2(11.8 \%)$ & $13(22.0 \%)$ & 0.554 \\
\hline $\mathrm{CHD}$ & $6(35.3 \%)$ & $9(15.3 \%)$ & 0.138 \\
\hline BMI & $23.12 \pm 3.13$ & $23.33 \pm 3.45$ & 0.822 \\
\hline UPDRS score & $76.06 \pm 16.80$ & $48.36 \pm 23.10$ & $<0.001$ \\
\hline Advanced stage & $15(88.2 \%)$ & $30(50.8 \%)$ & 0.006 \\
\hline BChE activity (IU/L) & $5974.96 \pm 1292.90$ & $7127.81 \pm 1253.21$ & 0.003 \\
\hline
\end{tabular}

PD, Parkinson's disease; SD, standard deviation; ALT, alanine transaminase; AST, aspartate aminotransferase; TC, total cholesterol; TG, triglyceride; CHD, coronary heart disease; BMI, body mass index; UPDRS, unified Parkinson's disease rating scale; BChE, butyrylcholinesterase.

TABLE 7: Univariate and multivariate binary logistic regression analysis of correlation factors for Parkinson's disease related dementia.

\begin{tabular}{|c|c|c|c|c|}
\hline \multirow{2}{*}{ Variable } & \multicolumn{2}{|c|}{ Univariate } & \multicolumn{2}{|c|}{ Multivariate } \\
\hline & OR (LL UL) & $P$ value & OR (LL UL) & $P$ value \\
\hline Disease duration & $1.154(1.033 \sim 1.290)$ & 0.011 & - & - \\
\hline Age & $1.080(1.006 \sim 1.159)$ & 0.034 & - & - \\
\hline Gender & $1.391(0.469 \sim 4.124)$ & 0.551 & l & l \\
\hline Albumin & $0.936(0.817 \sim 1.071)$ & 0.336 & l & l \\
\hline ALT & $1.009(0.992 \sim 1.026)$ & 0.302 & l & l \\
\hline AST & $1.018(0.991 \sim 1.046)$ & 0.197 & l & l \\
\hline TC & $0.680(0.343 \sim 1.349)$ & 0.270 & l & l \\
\hline TG & $0.758(0.339 \sim 1.694)$ & 0.499 & l & l \\
\hline BMI & $0.981(0.834 \sim 1.154)$ & 0.820 & l & l \\
\hline Smoking history & $1.508(0.406 \sim 5.593)$ & 0.539 & l & l \\
\hline Alcohol consumption & $2.946(0.590 \sim 14.708)$ & 0.188 & l & l \\
\hline Hypertension & $0.608(0.189 \sim 1.949)$ & 0.402 & l & l \\
\hline Diabetes mellitus & $0.850(0.163 \sim 4.439)$ & 0.847 & l & l \\
\hline Hypercholesterolaemia & $0.472(0.095 \sim 2.334)$ & 0.357 & l & l \\
\hline $\mathrm{CHD}$ & $3.030(0.893 \sim 10.284)$ & 0.075 & l & l \\
\hline Advanced stage & $7.250(1.522 \sim 34.543)$ & 0.013 & $5.612(1.132 \sim 27.827)$ & 0.035 \\
\hline BChE activity & $0.999(0.999 \sim 1.000)$ & 0.004 & $0.999(0.999 \sim 1.000)$ & 0.013 \\
\hline
\end{tabular}

OR, odds ratio; LL, lower limit; UL, upper limit; ALT, alanine transaminase; AST, aspartate aminotransferase; TC, total cholesterol; TG, triglyceride; BMI, body mass index; CHD, coronary heart disease; BChE, butyrylcholinesterase.

For the multivariate binary logistic regression analysis using a conditional backward method based on the results from univariate binary logistic regression analysis, the values were "I" if variables were excluded before the analysis, and the values were "-" if variables were excluded after the analysis. 
enough to discriminate between PD with dementia and patients without dementia.

In conclusion, BChE activity can be regarded as a biomarker in PD, especially in PD-related dementia. The same function has also been reported in other disorders, including Alzheimer's disease [11], vascular dementia [25], ischemic stroke [26], traumatic brain injury [7], postoperative delirium [27], severe trauma [28], septic shock [29], burn injuries [30], adverse cardiac events [16], hepatic disease [17], diabetes [31], and hyperlipidemia [32]. A former study on AChE levels in $\mathrm{PD}$ patients suggested that polymorphisms in $A C H E$ genes fail to initiate ACHE expression, resulting in an impaired Ach/DA balance and subsequent vulnerability of dopaminergic neurons and PD development [9]. The Ach/DA imbalance seemed to have difficulty in explaining the alteration of $\mathrm{BChE}$ activity in the other disorders. Some studies have also suggested that BChE activity reflects the intensity of cholinergic anti-inflammatory responses [7] or serves as a marker of changes in the sympathetic/parasympathetic balance [33]. The authors were also reluctant to explain the extensive involvement of BChE activity in multisystem disorders. BChE activity is repeatedly reported to be positively associated with TG, TC, BMI, and metabolic syndrome and is inversely associated with malnutrition [11], which can be also confirmed from our results. BChE is mainly synthesized in the liver and then released into the blood, suggesting that it could serve as a "nutrition protein." Levels decline with malnutrition due to disease or increase following overnutrition in metabolic syndrome. The potential pathophysiological mechanisms require further clarification. However, BChE activity could serve as a biomarker in $\mathrm{PD}$ patient to assess disease severity, discriminate PD-related dementia, evaluate efficacy of drugs, and instruct physicians about the course of clinical medications for dementia.

There were several limitations to this study. First, the number of included patients was limited and these results require further confirmation. This cohort study will continue and we hope it can also be validated by other research groups. Second, only BChE activity, without AChE activity, had been determined, as BChE activity was simple and convenient to obtain in our country.

\section{Conclusions}

Serum BChE activity, which decreased in PD patients and inversely correlated with disease severity, was clearly reported in PD patients for the first time. BChE activity could serve as a biomarker for detecting PD and related dementia in clinic.

\section{Conflicts of Interest}

The authors declare that they have no conflicts of interest.

\section{Authors' Contributions}

Mei-Xue Dong and You-Dong Wei conceived the study. The data analysis was performed by Mei-Xue Dong, Xiao-Min Xu, and Ling $\mathrm{Hu}$. The manuscript was revised by Mei-Xue Dong,
Yang Liu, and Yuan-Jun Huang. Mei-Xue Dong, Xiao-Min $\mathrm{Xu}$, and Ling $\mathrm{Hu}$ contributed equally to the manuscript.

\section{Acknowledgments}

This work was supported by the National Key Clinical Specialties Construction Program of China and Chongqing Science and technology commission (no. cstc2016jcy jA0423).

\section{References}

[1] S. Jesse, S. Lehnert, O. Jahn et al., "Differential sialylation of serpin Al in the early diagnosis of parkinson's disease dementia," PLoS ONE, vol. 7, no. 11, Article ID e48783, 2012.

[2] L. V. Kalia and A. E. Lang, "Parkinson's disease," The Lancet, vol. 386, no. 9996, pp. 896-912, 2015.

[3] I. Litvan, J. G. Goldman, A. I. Tröster et al., "Diagnostic criteria for mild cognitive impairment in Parkinson's disease: movement disorder society task force guidelines," Movement Disorders, vol. 27, no. 3, pp. 349-356, 2012.

[4] M. Emre, P. J. Ford, B. Bilgiç, and E. Y. Uç, "Cognitive impairment and dementia in Parkinson's disease: practical issues and management," Movement Disorders, vol. 29, no. 5, pp. 663-672, 2014.

[5] B. Singh and J. T. O’Brien, "When should drug treatment be started for people with dementia?" Maturitas, vol. 62, no. 3, pp. 230-234, 2009.

[6] J. C. Klein, C. Eggers, E. Kalbe et al., "Neurotransmitter changes in dementia with Lewy bodies and Parkinson disease dementia in vivo," Neurology, vol. 74, no. 11, pp. 885-892, 2010.

[7] Q.-H. Zhang, A.-M. Li, S.-L. He et al., "Serum total cholinesterase activity on admission is associated with disease severity and outcome in patients with traumatic brain injury," PLOS ONE, vol. 10, no. 6, Article ID e0129082, 2015.

[8] H.-Y. Chen, W. W.-J. Wang, C.-H. Chaou, and C.-C. Lin, "Prognostic value of serial serum cholinesterase activities in organophosphate poisoned patients," American Journal of Emergency Medicine, vol. 27, no. 9, pp. 1034-1039, 2009.

[9] L. Benmoyal-Segal, T. Vander, S. Shifman et al., "Acetylcholinesterase/paraoxonase interactions increase the risk of insecticideinduced Parkinson's disease," The FASEB Journal, vol. 19, no. 3, pp. 452-454, 2005.

[10] A. Berardelli, G. K. Wenning, A. Antonini et al., "EFNS/MDSES recommendations for the diagnosis of Parkinson's disease," European Journal of Neurology, vol. 20, no. 1, pp. 16-34, 2013.

[11] D. Dingova, T. Fazekas, P. Okuliarova, J. Strbova, M. Kucera, and A. Hrabovska, "Low plasma cholinesterase activities are associated with deficits in spatial orientation, reduced ability to perform basic activities of daily living, and low body mass index in patients with progressed alzheimer's disease," Journal of Alzheimer's Disease, vol. 51, no. 3, pp. 801-813, 2016.

[12] L. Hu, M.-X. Dong, H. Zhao, G.-H. Xu, and X.-Y. Qin, "Fibulin5: a novel biomarker for evaluating severity and predicting prognosis in patients with acute intracerebral haemorrhage," European Journal of Neurology, vol. 23, no. 7, pp. 1195-1201, 2016.

[13] M.-X. Dong, Q.-C. Hu, P. Shen et al., "Recombinant tissue plasminogen activator induces neurological side effects independent on thrombolysis in mechanical animal models of focal cerebral infarction: A systematic review and meta-analysis," PLoS ONE, vol. 11, no. 7, Article ID e0158848, 2016. 
[14] M. Tinazzi, G. Abbruzzese, A. Antonini et al., "Reasons driving treatment modification in Parkinson's disease: results from the cross-sectional phase of the REASON study," Parkinsonism and Related Disorders, vol. 19, no. 12, pp. 1130-1135, 2013.

[15] M. Zhang, R. Katzman, D. Salmon et al., "The prevalence of dementia and Alzheimer's disease in Shanghai, China: impact of age, gender, and education," Annals of Neurology, vol. 27, no. 4, pp. 428-437, 1990.

[16] Y. Arbel, S. Shenhar-Tsarfaty, N. Waiskopf et al., "Decline in serum cholinesterase activities predicts 2-year major adverse cardiac events," Molecular Medicine, vol. 20, pp. 38-45, 2014.

[17] O. O. Ogunkeye and A. I. Roluga, "Serum cholinesterase activity helps to distinguish between liver disease and non-liver disease aberration in liver function tests," Pathophysiology, vol. 13, no. 2, pp. 91-93, 2006.

[18] L. Santarpia, I. Grandone, F. Contaldo, and F. Pasanisi, "Butyrylcholinesterase as a prognostic marker: a review of the literature," Journal of Cachexia, Sarcopenia and Muscle, vol. 4, no. 1, pp. 3139, 2013.

[19] E. W. Randell, M. S. Mathews, H. Zhang, J. S. Seraj, and G. Sun, "Relationship between serum butyrylcholinesterase and the metabolic syndrome," Clinical Biochemistry, vol. 38, no. 9, pp. 799-805, 2005.

[20] A. J. Noyce, J. P. Bestwick, L. Silveira-Moriyama et al., "Metaanalysis of early nonmotor features and risk factors for Parkinson disease," Annals of Neurology, vol. 72, no. 6, pp. 893-901, 2012.

[21] E. H. Sklan, A. Lowenthal, M. Korner et al., "Acetylcholinesterase/paraoxonase genotype and expression predict anxiety scores in health, risk factors, exercise training, and genetics study," Proceedings of the National Academy of Sciences of the United States of America, vol. 101, no. 15, pp. 5512-5517, 2004.

[22] V. Dhananjayan, B. Ravichandran, N. Anitha, and H. Rajmohan, "Assessment of acetylcholinesterase and butyrylcholinesterase activities in blood plasma of agriculture workers," Indian Journal of Occupational and Environmental Medicine, vol. 16, no. 3, pp. 127-130, 2012.

[23] L. Kapka-Skrzypczak, K. Sawicki, M. Czajka, W. A. Turski, and M. Kruszewski, "Cholinesterase activity in blood and pesticide presence in sweat as biomarkers of children's environmental exposure to crop protection chemicals," Annals of Agricultural and Environmental Medicine, vol. 22, no. 3, pp. 478-482, 2015.

[24] G. Kleiner-Fisman, M. B. Stern, and D. N. Fisman, "Healthrelated quality of life in Parkinson disease: correlation between health utilities index III and unified Parkinson's disease rating scale (UPDRS) in U.S. male veterans," Health and Quality of Life Outcomes, vol. 8, article no. 91, 2010.

[25] Y. Xiao, Z.-Z. Guan, C.-X. Wu, Y. Li, S.-X. Kuang, and J.-J. Pei, "Correlations between cholinesterase activity and cognitive scores in post-ischemic rats and patients with vascular dementia," Cellular and Molecular Neurobiology, vol. 32, no. 3, pp. 399407, 2012.

[26] E. B. Assayag, S. Shenhar-Tsarfaty, K. Ofek et al., "Serum cholinesterase activities distinguish between stroke patients and controls and predict 12-month mortality," Molecular Medicine, vol. 16, no. 7-8, pp. 278-286, 2010.

[27] J. Cerejeira, P. Batista, V. Nogueira, H. Firmino, A. Vaz-Serra, and E. B. Mukaetova-Ladinska, "Low preoperative plasma cholinesterase activity as a risk marker of postoperative delirium in elderly patients," Age and Ageing, vol. 40, no. 5, Article ID afr053, pp. 621-626, 2011.
[28] L. Ba, D.-Q. Wu, A.-Y. Qian, M. Zhang, and B. Xiong, "Dynamic changes of serum cholinesterase activity after severe trauma," Journal of Zhejiang University: Science B, vol. 15, no. 12, pp. 10231031, 2014.

[29] M. Bahloul, N. Baccouch, K. Chtara et al., "Value of serum cholinesterase activity in the diagnosis of septic shock due to bacterial infections," Journal of Intensive Care Medicine, vol. 32, no. 5, pp. 346-352, 2016.

[30] L.-P. Kamolz, H. Andel, M. Greher, D. Andel, G. Meiss, and M. Frey, "Serum cholinesterase activity in patients with burns," Clinical Chemistry and Laboratory Medicine, vol. 40, no. 1, pp. 60-64, 2002.

[31] G. Inácio Lunkes, F. Stefanello, D. Sausen Lunkes, V. Maria Morsch, M. R. C. Schetinger, and J. F. Gonçalves, "Serum cholinesterase activity in diabetes and associated pathologies," Diabetes Research and Clinical Practice, vol. 72, no. 1, pp. 28-32, 2006.

[32] J. Kálmán, A. Juhász, Z. Rakonczay et al., "Increased serum butyrylcholinesterase activity in type IIb hyperlipidaemic patients," Life Sciences, vol. 75, no. 10, pp. 1195-1204, 2004.

[33] S. Shenhar-Tsarfaty, S. Berliner, N. M. Bornstein, and H. Soreq, "Cholinesterases as biomarkers for parasympathetic dysfunction and inflammation-related disease," Journal of Molecular Neuroscience, vol. 53, no. 3, pp. 298-305, 2014. 


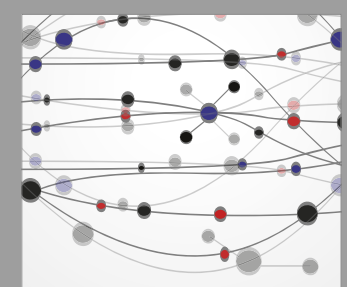

The Scientific World Journal
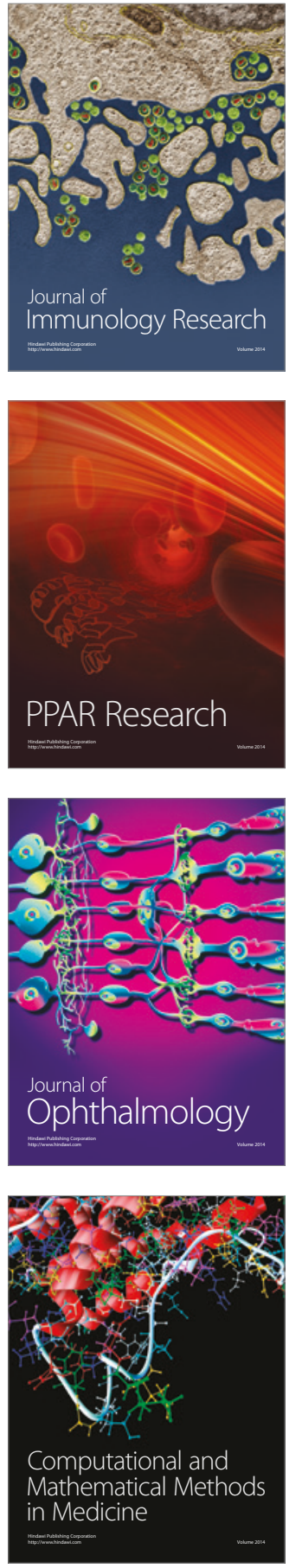

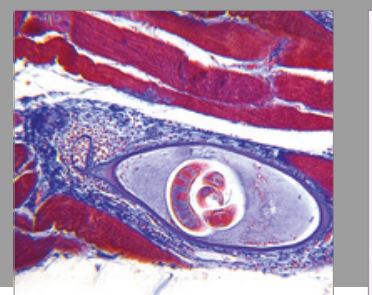

Gastroenterology Research and Practice
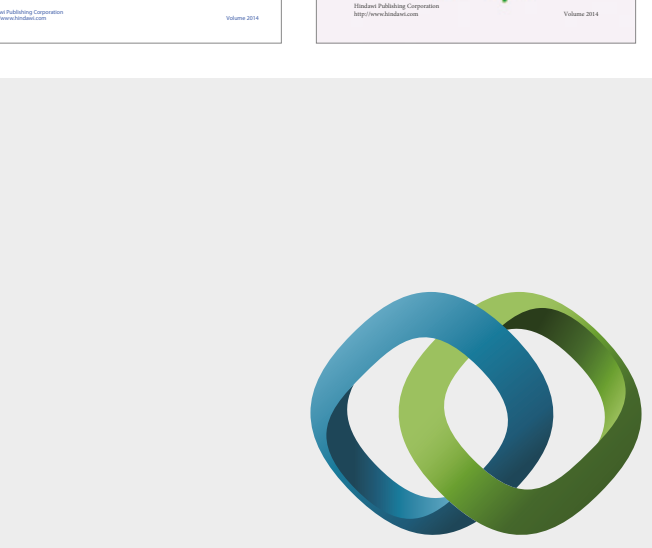

\section{Hindawi}

Submit your manuscripts at

https://www.hindawi.com
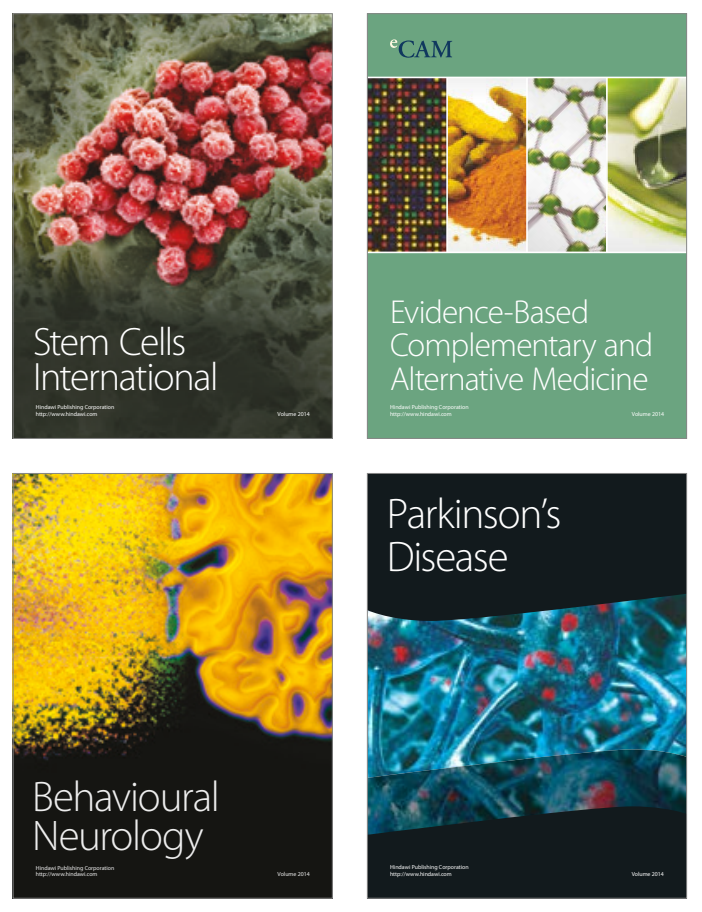
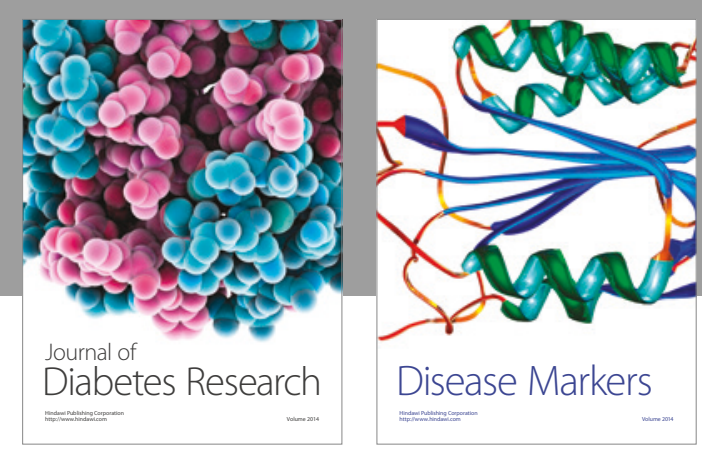

Disease Markers
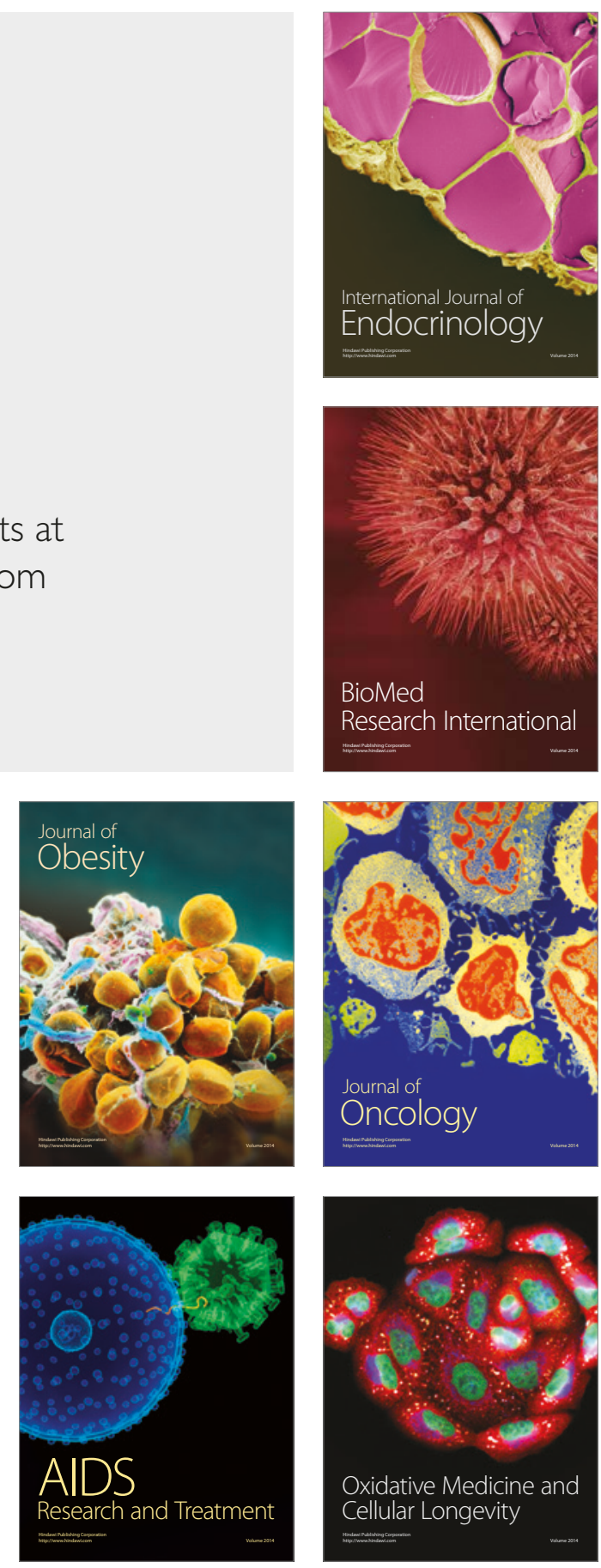